\title{
Planning omni-present networks of the future
}

\author{
Covering the smart city with a ubiquitous network fabric
}

\author{
Jonathan Spruytte, Bram Naudts, Koen Casier, Jan Van Ooteghem, Sofie Verbrugge \\ Ghent University - iMinds \\ Internet Based Communication Networks and Services research group (IBCN) \\ Gaston Crommenlaan 8 bus 201, B-9050 Gent, Belgium \\ Email: \{jonathan.spruytte, bram.naudts, koen.casier, jan.vanooteghem, sofie.verbrugge\}@intec.ugent.be
}

\begin{abstract}
On one hand, customers expect mobile broadband at any time and place at increasing bandwidth while on the other hand there is an evolution towards smart cities which include video monitoring and large amount of extra sensors putting additional strain on the existing network. Because of this, optimal long term network planning is of crucial importance. This planning has to take into account the ever increasing bandwidth demand and user adoption. In this publication, a genetic algorithm is proposed to find the optimal location of base stations to achieve a full coverage. Using this algorithm, a clear comparison has been made between a short-term (incremental) and a long-term (anticipated) planning.
\end{abstract}

Keywords— network rollout; wireless network planning

\section{The role of an omni-present network in the smart city}

Bandwidth to any device is increasing and people are willing to pay to connect to rich media at all times. Recent evolutions in wireless networks open up higher bandwidth connections to the customer and the battle for customers has been recently focused on this aspect. Next to the end customers, smart cities are also a great driver for bandwidth, as many smart monitoring and feedback applications could greatly benefit from a high bandwidth and reliable connections [1][2].

With the advent of sensor networks, cloud computing and wearable end devices, applications require to be constant online and have a sufficient bandwidth available. Many novel services target the customer end device and send dedicated data to these customers, but bandwidth demand is not limited to this server-to-client push or pull communication. As sensor maps and cloud computing demand bandwidth to push data quickly from input up to calculation and knowledge generation.

Cities, transportation lines and hubs are, next to the traditional office and home environments, hotspots of these demands for bandwidth. While at home and at work, one or a limited amount of access points could suffice, a city wide network will require an area covering set of access points, providing sufficient bandwidth to customers in reach. An optimal tuning of this network is required and the first task to tackle is determining the amount and location of wireless access points to put in the network, in order to cover a full transportation line and/or part of a city. This will enable novel applications for people on the go, for machines on the road or track, detailed transportation monitoring, etc. all leading to the emergence of a true smart city.

While the smart city is all about applications, clearly moving towards the smart city is about data, devices and linking these together. In this chicken-egg problem, the availability of an omni-present network triggered by a push from transportation and municipal investments could well be the stepping stone towards novel applications. The way to get there is to find the optimal match between costs, coverage and bandwidth for a continuously increasing bandwidth and connectivity demand. This paper focuses on developing a solid algorithmic basis for determining the optimal network rollout technique for such omni-present smart city network.

The remainder of this paper is structured as follows: In section II the planning problem is further discussed after which the genetic algorithm is described together with two rollout techniques. Next in section III the two techniques are applied to two real life cases. Finally section IV summarizes this paper and proposes possible tracks for future work.

\section{Long term wireless network planning}

Wireless networks are evolving technology wise e.g. the transition from $3 \mathrm{G}$ towards LTE (Long-Term Evolution). These network upgrades open up higher bandwidths, typically combined with a smaller cell sizes [3]. Clearly with a growth of bandwidth or an upgrade of the network, extra antennas will be required. Adding these on demand is the most straightforward approach, however this will lead to a very fragmented and suboptimal network in the future. On the other hand, installations with a view on the future will gradually lead to a less fragmented and more optimal installation.

\section{A. Estimating cell size with path-loss models}

In order to create an optimal long term wireless network planning, it is important to get an estimation of the range of a base station. To do so, the link budget has to be determined. The link budget takes all the gains and the losses that occur during the propagation through the medium, from transmitter 
to the receiver, into account. The link budget is needed to calculate the maximum allowable path loss $\mathrm{PL}_{\max }$ (in $\mathrm{dB}$ ) to which a transmitted signal can be subjected while still being detectable by a receiver. To determine $\mathrm{PL}_{\max }$, the gain and loss parameters of Table 1 are taken into account. These parameters are retrieved from [4] and represent parameters retrieved from specifications and typical values proposed by the operators themselves for LTE.

\begin{tabular}{|l|l|l|}
\hline Parameter & LTE & Unit \\
\hline Input power of base station & 43 & $\mathrm{dBm}$ \\
\hline Antenna gain of base station & 18 & $\mathrm{dBi}$ \\
\hline Antenna gain of mobile station & 0 & $\mathrm{dBi}$ \\
\hline Number of MIMO Tx antennas & 1,2 & - \\
\hline Rx antennas & 1,2 & - \\
\hline MIMO gain & $\begin{array}{l}1 \times 1: 0 \\
2 \times 2: 3.5\end{array}$ & $\mathrm{~dB}$ \\
\hline $\begin{array}{l}\text { Cyclic combining gain of base } \\
\text { station }\end{array}$ & 3 & $\mathrm{~dB}$ \\
\hline Bandwidth & 5 & $\mathrm{MHz}$ \\
\hline Receiver SNR & {$[-1.5310 .514$} & $\mathrm{dB}$ \\
& $192329.4]^{1}$ & \\
\hline Number of used subcarriers & 301 & - \\
\hline Number of total carriers & 512 & - \\
\hline
\end{tabular}

Table 1: Link budget parameters for LTE

Once the maximum allowable path loss $\mathrm{PL}_{\max }$ is known, the maximum cell size (in meters) covered by an LTE base station can be determined using a path loss model. These take into account $\mathrm{PL}_{\max }$, the shadowing margin, the frequency, the height of the base station and the height of the receiver. Further, when calculating for a moving vehicle, we apply a Doppler margin of $3 \mathrm{~dB}$ [6] in order to take speeds up to 150 $\mathrm{km} / \mathrm{h}$ into account.

Here, the Erceg C path loss model [7] is assumed. This model is based on experimental data in different suburban areas. Erceg categorizes three different terrain categories: category A corresponds to moderate-to-heavy tree density and has the highest path loss, category B corresponds to a mostly flat terrain with moderate-to-heavy tree densities or a hilly terrain with low tree density, category $\mathrm{C}$ corresponds to a flat terrain with light tree density and has the minimum path loss. For this publication, the model has been set fixed to Erceg $\mathrm{C}$ in compliance with other publications [8]-[10]. Different models, depending on the situation ( $\mathrm{C}$ for transportation lines and A or B for a more urban area) are possible too. Finally, Table 2 summarizes the remaining configuration parameters used in this article.

\begin{tabular}{|l|l|l|}
\hline Parameter & LTE & Unit \\
\hline Frequency & 900 & $\mathrm{MHz}$ \\
\hline Height of base station & 30 & $\mathrm{~m}$ \\
\hline Height of receiver & 1,5 & $\mathrm{~m}$ \\
\hline Doppler margin & $0-3$ & $\mathrm{~dB}$ \\
\hline
\end{tabular}

Table 2: Network configuration table

1 [1/3 QPSK, 1/2 QPSK, 2/3 QPSK, 1/2 16-QAM, 2/3 16-QAM, 1/2 64-QAM, 2/3 64QAM][5].

\section{B. Wireless network planning problem}

Within a wireless network planning problem, also known as the radio network design problem, an optimal coverage of an area has to be determined. Optimal means as close to the targeted goal as possible (typically between $90 \%$ and $100 \%$ ) while using as few antenna locations as possible. This problem is strongly related to the circle packing [11] and the bin packing problem [12] and can also be classified as an NP-hard problem itself. Since this problem is a NP-hard problem, more advanced heuristics are required to find an optimal solution; in this case a genetic algorithm has been developed.

Genetic algorithms are search algorithms, which use techniques found in natural evolution. Within the algorithm, the group of possible solutions (the population) evolves generation after generation, using three basic actions: survival of the fittest (selection), crossover and mutation. Selection ensures that the best solutions are selected to breed offspring (using crossover) and in the meantime that the worse solutions are removed from the population. Finally mutation slightly changes the solutions in an attempt to improve them.

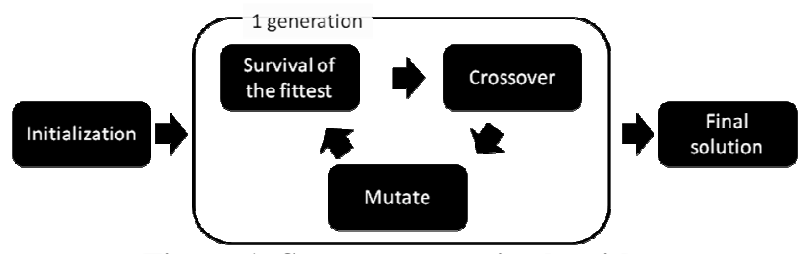

Figure 1: Structure genetic algorithm

One of the core elements of a genetic algorithm is the fitness function, which calculates a fitness (quality) value for each individual of the population. The fitness function is highly dependent on the problem. Equation 1 shows the proposed formula to evaluate the fitness of a solution based on the total coverage and number of antennas and has earlier been stated in [13] and [14].

$$
\text { fitness }=\frac{\text { coverage }^{2}}{\# \text { base stations }}
$$

\section{Equation 1: Fitness function taken into account coverage and number of base stations}

This fitness function could be adapted to take into account other parameters e.g. the total length of cable to connect all base stations locations or the total power usage of all the base stations. In [15] a more in-depth overview of the techniques and capabilities of genetic algorithms is provided in combination with the mathematical background and a number of other evolutionary methodologies..

\section{Yearly incremental vs anticipated rollout approach}

Any network can be rolled out using two techniques. On one hand there is the yearly incremental installation method. Here, every time an upgrade is required, the best solution for the current situation is chosen, well aware this will not yield the best results in the future. On the other hand an anticipated rollout can be used [16]. This kind of rollout defines a specific goal for a number of years in the future. Once this goal is defined, a solution is searched for each year taking into 
account the long term goal. The downside to this technique is often a sub-optimal solution in the years leading towards the goal. Similar conclusions were made for a FTTH (Fiber to the home) case in [17].

When applying these rollout techniques to the radio design problem, we expect the following. Using the incremental rollout, every time a higher bandwidth is required, new base station locations are added to achieve an optimal coverage. Selecting the locations on a year by year basis means more antennas will have been installed in the end than actually required. When using the anticipated rollout, a bandwidth goal is set for a number of years in the future for which an optimal coverage is searched. When a higher bandwidth has is required, only base station locations of the final goal are taken into account. This ensures an optimal positioning of the base stations in the last year.

Both approaches take into account base stations that have been installed in the previous periods. In the same manner, the algorithm can be used to take into account base stations that have been installed in a real life case. This way not only initial Greenfield situations, like the ones discussed further in this paper but also Brownfield situations with real operator data can be simulated.

\section{Smart City and Smart Transportation use cases}

People expect high bandwidths and reliable networks at any place and time e.g. video streaming on the train [18]. On the other hand, the introduction of smart cities, which include a high number of connected sensors put extra load on the networks. Both of these scenarios have been elaborated in two use cases. First a specific transport track has been considered, in this case a railroad, discussed in section A. Next, in section B the optimal coverage of a square has been determined, which could be a (part of a) city. For these two cases, both rollout approaches have been calculated for 5 periods, using the bandwidth steps given in Table 3 .

\begin{tabular}{|c|c|}
\hline Period & $\begin{array}{c}\text { Bandwidth } \\
\text { (Mbps) }\end{array}$ \\
\hline $\mathbf{1}$ & 2.8 \\
\hline $\mathbf{2}$ & 4.2 \\
\hline $\mathbf{3}$ & 5.7 \\
\hline $\mathbf{4}$ & 8.5 \\
\hline $\mathbf{5}$ & 11.3 \\
\hline
\end{tabular}

Table 3: Bandwidth goal per period overview

\section{A. Omni-present networks connecting transportation hubs and tracks in Belgium}

The first considered case is the train connection going from Ostend to Eupen and is based upon GIS-data received from OpenStreetMap [19] (the data has been rotated slightly for the ease of comparison). For both rollout situations the coverage goal has been set to $100 \%$.

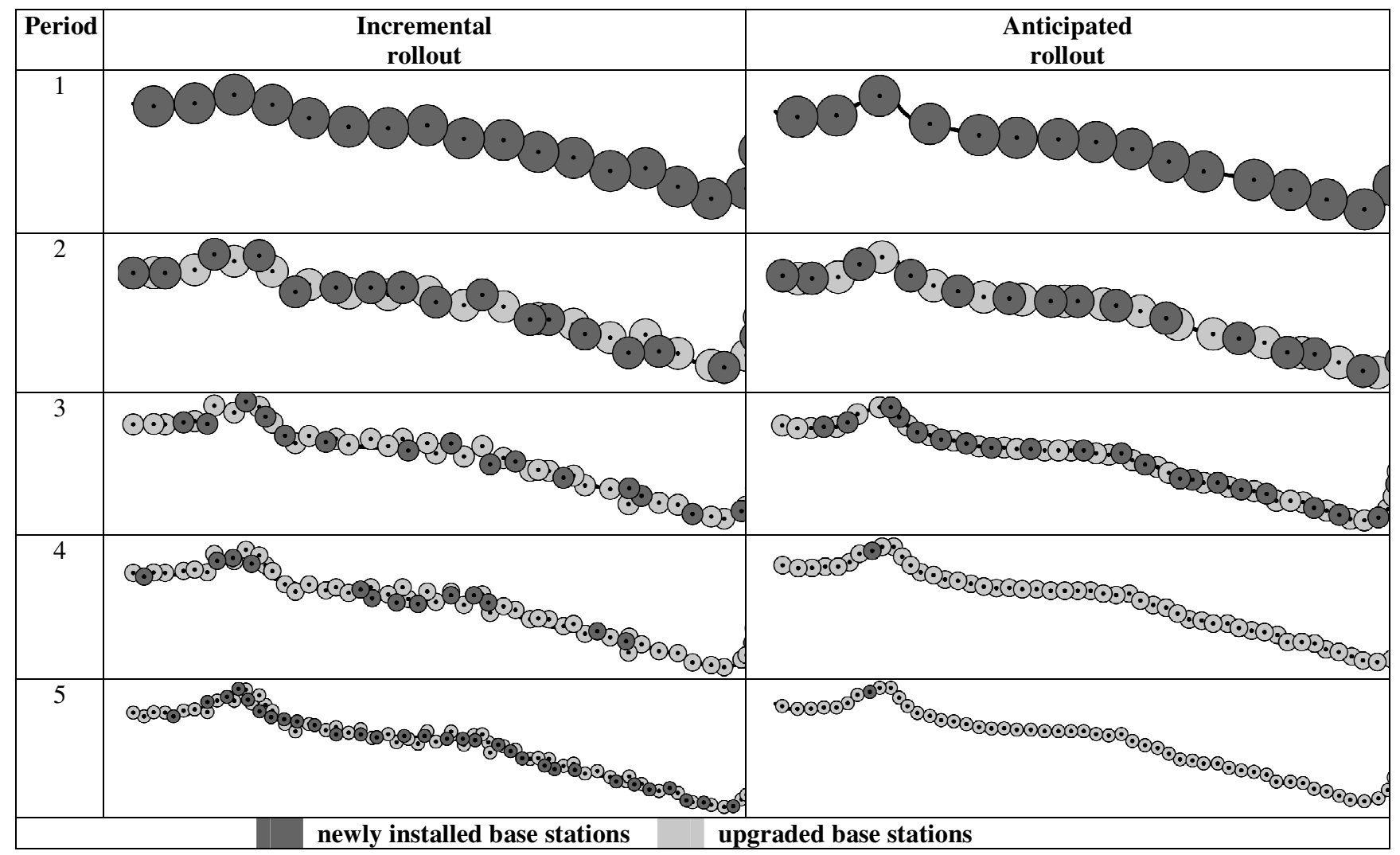

Figure 2: Base stations along train line incremental vs. anticipated rollout (only Ostend - Brussels) shown 


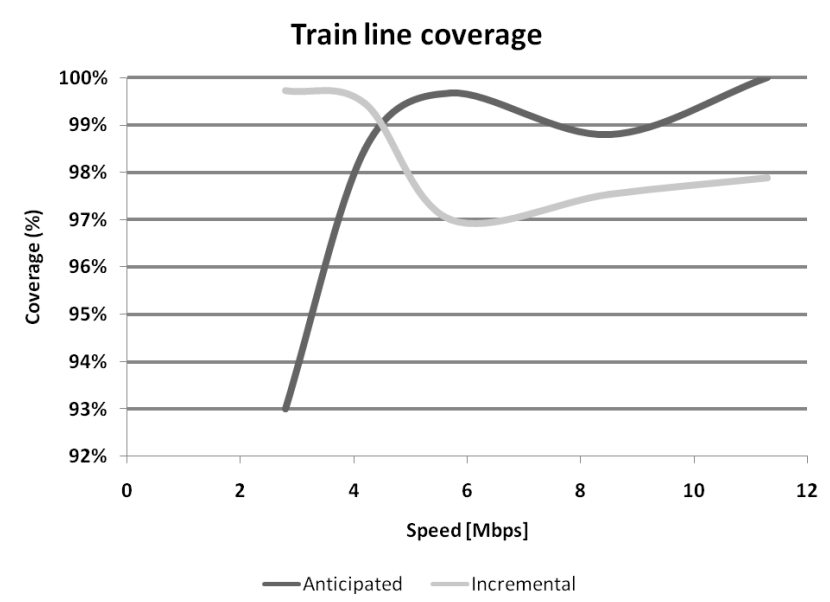

Figure 3: Train line rollout comparison - coverage (only top $8 \%$ shown)

As clearly derived from Figure 4, an incremental rollout for a total of 5 time periods results in $70 \%$ more base stations compared to an anticipated rollout. Important to notice is that the anticipated rollout clearly has a suboptimal solution in the first period, while the incremental approach has the same problem in the last periods. Furthermore, in the second period (4.2Mbp) the solution of both methods is almost identical. In the third period $(5.8 \mathrm{Mbps})$, the incremental method uses a few base stations less than the anticipated approach; this is because the many small coverage gaps, as visible in Figure 2, are not worth covering when using the earlier mentioned fitness function; this clearly results in a lower coverage for the incremental approach as reflected in Figure 3.

\section{B. Planning city wide wireless networks}

In the second case, a square of $25 \mathrm{~km}^{2}$ has to be covered and this again using the incremental and anticipated methods. This second case is obviously harder to optimize, as this is a two-dimensional optimization, while the case in section A was almost a one-dimensional problem. However, here again, the anticipated rollout method clearly results in a $60 \%$ better outcome as deducted from Figure 6. Similar to case A, the anticipated method has a lower coverage in the first years (Figure 5). In contrast to case A, both solutions for the second step (4.2Mbps) no longer result in the coverage. Further, in case A the incremental method was slightly better for the third period, while here this is clearly not the case, as the anticipated method uses fewer base stations throughout the entire rollout towards the final goal.
Train line coverage: number of base stations

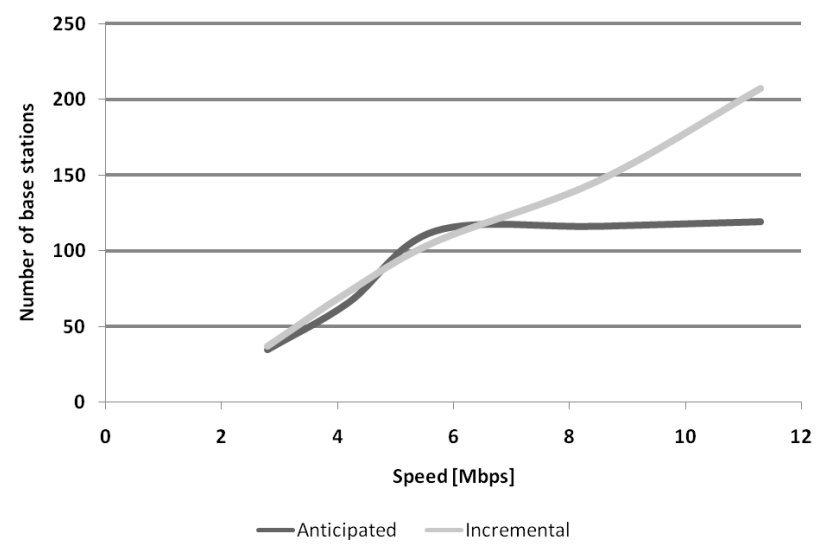

Figure 4: Train line rollout comparison - required base stations

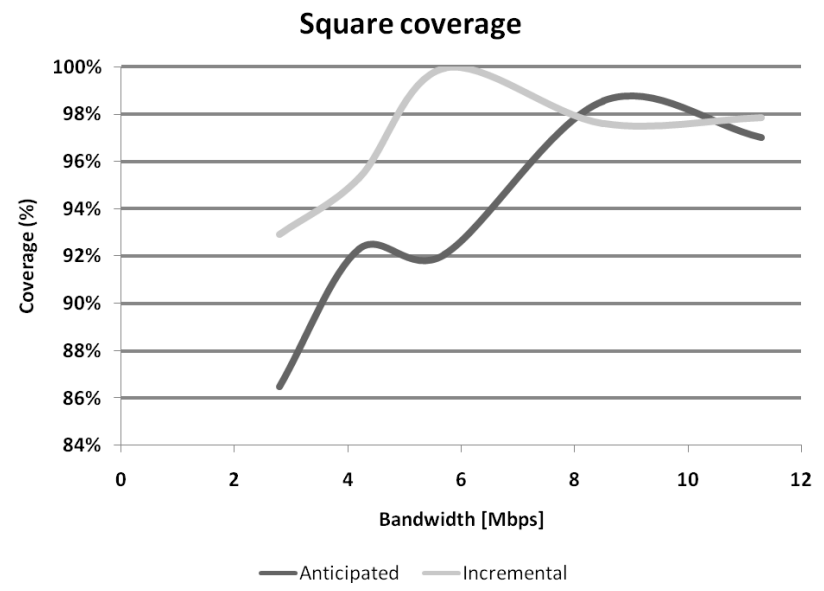

Figure 5: Square rollout comparison - coverage (only top $16 \%$ shown)

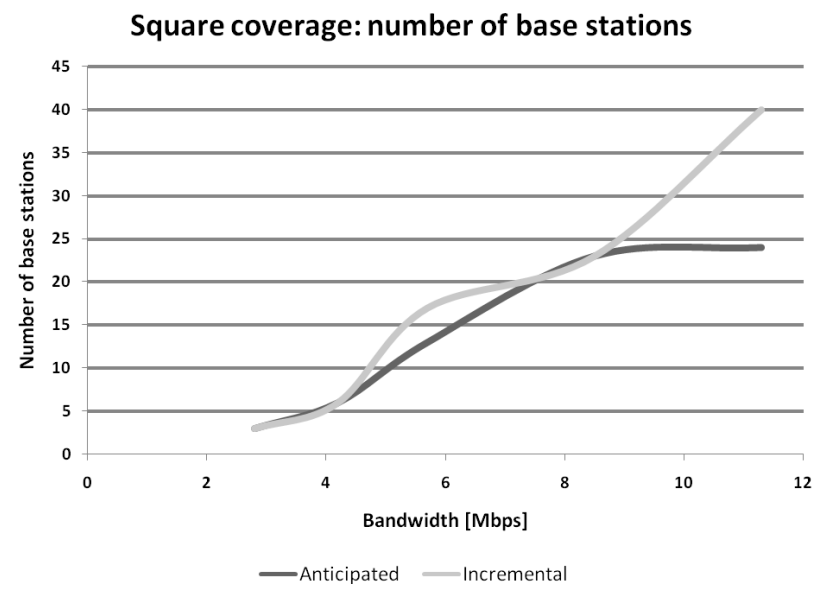

Figure 6: Square rollout comparison - required base stations 


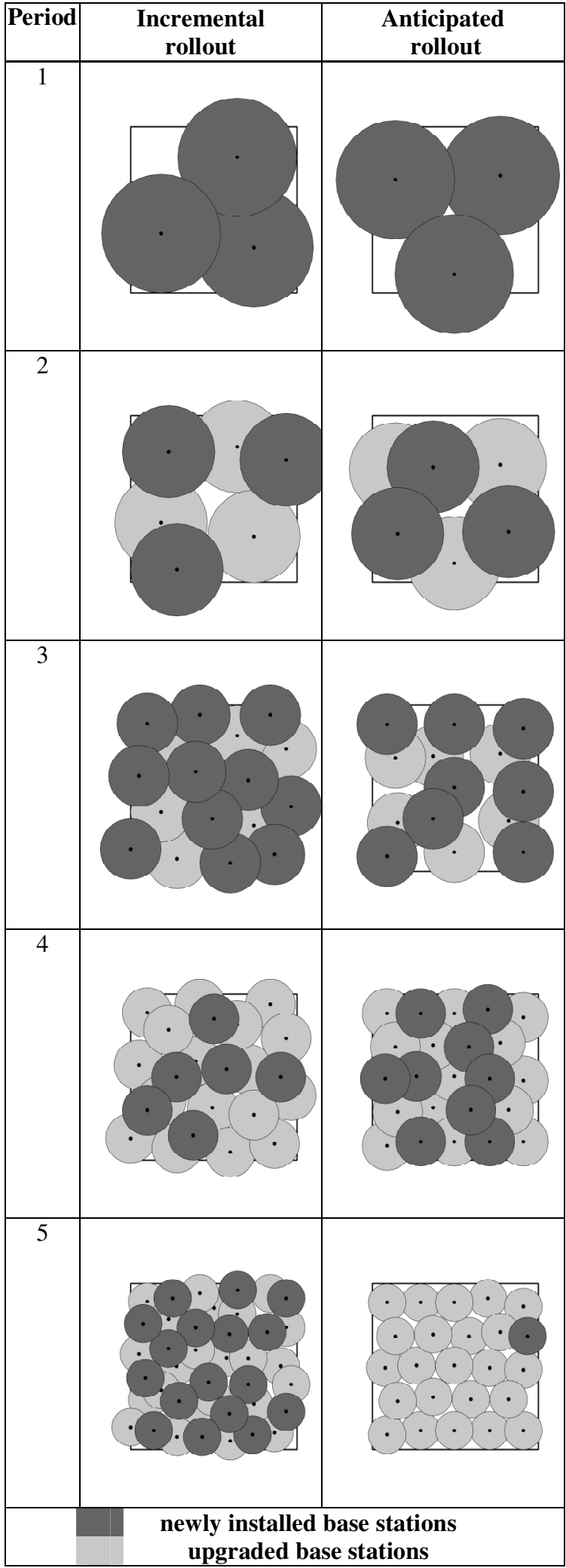

Figure 7: Base stations in city area incremental vs. anticipated rollout

\section{Iv. Conclusions and Future work}

With the rise of smart connected end-devices, the bandwidth demand for the network is increasing incessantly. To keep up with the ever growing user adoption and bandwidth requirements, a static network planning is no longer viable. The wireless network has to grow to meet these demands, and new base stations have to be installed to match this growth. Finding the best way to grow a network over many years is a complex economic question. In this publication, an optimal planning algorithm for such wireless network growth is constructed and investigated. This algorithm anticipates the growth over several years and optimally positions new wireless base stations in early stages at locations that will be required at a later stage. A comparison between such an anticipated planning and a more shortsighted incremental planning has been made. This comparison has pointed out that by creating a long-term anticipated planning, up to $60 \%$ less base stations are required to obtain an optimal coverage compared to an incremental planning. Clearly anticipating the future network in new installations is an economic valuable approach.

In the future, this analysis will be extended in a number of ways. Firstly, larger cases like the entire train network in Flanders or Belgium or Brownfield cases were the existing $3 \mathrm{G}$ network is upgraded towards LTE will be considered. Also the 2D planning of wireless network evolution will be focus of further study. Understanding and extending algorithms for optimal positioning of wireless base stations in city centers and along public transportation lines is an important aspect of building the network fabric of the future. Secondly, this approach can also be applied to totally different cases; either to other types of networks e.g. sensor networks or networks in which additional parameters need optimizing e.g. the total amount of cable to connect all base stations or the total power usage of the network. Lastly, the influence of changing essential network parameters (e.g. the frequency) during the rollout will be tested. This way the fast and ever-changing technological evolution can be fully included in the proposed methodology.

\section{Acknowledgment}

This research was carried out as part of the iMinds RAILS project. This project is co-funded by iMinds, a research institute founded by the Flemish Government. Companies and organizations involved in the project are Televic Rail, Option NV, Bombardier Transportation, Belgacom, Uify, NMBS, with project support of IWT.

\section{References}

[1] Bram Naudts, Jan Van Ooteghem, Bart Lannoo, Sofie Verbrugge, Didier Colle, Mario Pickavet, On the right tracks? Continuous broadband Internet on trains, ITP, Vol. 7 Part 12013.

[2] T. Evens, D. Schuurman, L. D. Marez, and G. Verleye, "Forecasting broadband internet adoption on trains in Belgium," Telematics and Informatics, vol. 27, no. 1, pp. $10-20,2010$. 
[3] Yamao, Y.; Suda, H.; Umeda, N.; Nakajima, N., "Radio access network design concept for the fourth generation mobile communication system," Vehicular Technology Conference Proceedings, 2000. VTC 2000-Spring Tokyo. 2000 IEEE 51st, vol.3, no., pp.2285,2289 vol.3, 2000

[4] Deruyck, Margot, Emmeric Tanghe, Wout Joseph, and Luc Martens. 2011. Modelling and Optimization of Power Consumption in Wireless Access Networks. Computer Communications 34 (17): 2036 - 2046.

[5] Basit, Abdul. "Syed-" Dimensioning of LTE Network: Description of Models and Tool." Coverage and Capacity Estimation of 3GPP Long Term Evolution radio interface" Masters Thesis submitted in Helsink University of Technology.

[6] IEEE Std 802.16e/D12, Draft IEEE Standard for Local and metropolitan area networks, Part 16: Air Interface for Fixed and Mobile Broadband Wireless Access Systems

[7] Erceg, Vinko, et al. "An empirically based path loss model for wireless channels in suburban environments." Selected Areas in Communications, IEEE Journal on 17.7 (1999): 1205-1211.

[8] Simon Evenepoel, Jan Van Ooteghem, Bart Lannoo, Sofie Verbrugge, Mario Pickavet, Didier Colle, Municipal Wi-Fi deployment and crowd-sourced strategies, Journal of the institute of telecommunications professionals, Vol. 7, Part 1, pp. 24-29, 2013

[9] Erik Vanhauwaert, Sofie Verbrugge, Wim Vandenberghe, Mario Pickavet, Piet Demeester, Realistic Cost Estimation of an intelligent transportation system roll-out, The International Journal of the Telecom Professional (ITP), Vol. 5 Part 1201

[10] Bram Naudts, Jonathan Spruytte, Jan Van Ooteghem, Sofie Verbrugge, Didier Colle, Mario Pickavet, Internet on trains: A multicriteria ananlysis of on-board deployment options for on-train cellular connectivity, Networks 2014

[11] Stephenson, Kenneth. Introduction to Circle Packing : the Theory of Discrete Analytic Functions. New York: Cambridge University Press, 2005.

[12] Andrea Lodi, Silvano Martello, Daniele Vigo, Recent advances on two-dimensional bin packing problems, Discrete Applied Mathematics, Volume 123, Issues 1-3, 15 November 2002, Pages 379-396

[13] Alba. Evolutionary Algorithms for Optimal Placement of Antennae in Radio Network Design, IEEE Proceedings of NIDISC'04, Santa Fe, New Mexico, USA, pp. 168, 2004.

[14] Silvio Priem Mendes, Juan A. Gomez Pulido, Miguel A. Vega Rodriguez, Maria D. Jaraiz Simon, Juan M. Sanchez Perez, "A Differential Evolution Based Algorithm to Optimize the Radio Network Design Problem," 2012 IEEE 8th International Conference on E-Science, p. 119, Second IEEE International Conference on eScience and Grid Computing (e-Science'06), 2006

[15] Whitley, D. A genetic algorithm tutorial. Tech. rep. CS-93-103, Colorado State University, 1993

[16] S. Verbrugge, D. Colle, M. Pickavet, P. Demeester, Anticipating on future evolutions during the network planning process, OFC 2003 Optical Fiber Communication Conference, ISBN 1-55752-731-8, Vol. 1, 23-28 March 2003, Atlanta, Georgia, USA, pp. 415-416

[17] Jan Van Ooteghem, Koen Casier,Luc De Heyn, Raf Meersman, Bart Rosseau, Didier Colle, Mario Pickavet, "Municipal driven fiber access network rollout", Paper in the proceedings of the 19th ITS Biennial Conference, 18-21 November 2012, Bangkok, Thailand.

[18] Bram Naudts, Jan Van Ooteghem, Sofie Verbrugge, Didier Colle, Mario Pickavet, Insights in the cost of continuous broadband internet on trains for multi-service deployments by multiple actors with resource sharing, EURASIP Journal on Wireless Communications and Networking

[19] OpenSteetMap.org 